\title{
Multipseudopotential interaction: A solution for thermodynamic inconsistency in pseudopotential lattice Boltzmann models
}

\author{
Sorush Khajepor, ${ }^{1}$ John Wen, ${ }^{2}$ and Baixin Chen ${ }^{1, *}$ \\ ${ }^{1}$ School of Engineering and Physical Sciences, Heriot-Watt University, Edinburgh EH14 4AS, United Kingdom \\ ${ }^{2}$ Department of Mechanical and Mechatronics Engineering, University of Waterloo, 200 University Avenue West, \\ Waterloo, Ontario N2L 3G1, Canada
}

(Received 17 December 2013; revised manuscript received 3 October 2014; published 2 February 2015)

\begin{abstract}
Pseudopotential lattice Boltzmann (LB) models have been recognized as efficient numerical tools to simulate complex fluid systems, including those at thermodynamic equilibrium states and with phase transitions. However, when the equation of state (EOS) of real fluids is implemented, the existing pseudopotential LB models suffer from thermodynamic inconsistency. This study presents a multipseudopotential interparticle interaction (MPI) scheme, which is fully consistent with thermodynamics and applicable to engineering applications. In this framework, multiple pseudopotentials are employed to represent dominant interaction potentials at different extents of the mean free path of particles. By simulating van der Waals and Carnahan-Starling fluids, it is demonstrated that the MPI scheme can correctly simulate the physical nature of two-phase systems on the lattice including the continuum predictions of liquid-vapor coexistence states and the sound speeds in liquid and vapor phases. It is also shown that the lattice interactions of the MPI scheme represent underlying molecular interactions as they vary in a broad range from strong short-distance repulsions to weak long-distance attractions during phase transitions. Consequently, the MPI is proved to be a reliable LB scheme as it avoids generating unphysical potentials in implementing the EOSs of real fluids and limiting the spurious velocities at the interface of two-phase systems. Additionally, a straightforward procedure is suggested and discussed to preset the MPI system with the two-phase properties of a selected fluid.
\end{abstract}

DOI: 10.1103/PhysRevE.91.023301

PACS number(s): 47.11.- j, 05.70.Ce, 47.55.-t

\section{INTRODUCTION}

The lattice Boltzmann equation (LBE) was originally developed for ideal gases. Further efforts have been made to describe the nonideal gases (i.e., real fluids) and model the interfacial interactions of multiphase or multicomponent flows. Among these proposed approaches [1-8], Shan and Chen's (SC) model $[2,3]$ has been extensively used to study two-phase flows, largely due to its straightforward definition and simple implementation [9-16]. The principle of this model lies in the incorporation of a long-range interparticle interaction function (pseudopotential) into the LBE. A major concern about the SC model arises from its thermodynamic inconsistency caused by the discretization effect of the lattice. The simulated systems recover slightly different liquid and vapor densities in comparison with analytical solutions obtained from the Maxwell equal-area rule. This discrepancy increases as the temperature decreases and recedes from the critical point. The deviation indicates a lack of energy conservation in the SC model [17].

The aforementioned issue was first noticed by Shan and Chen [3], and the pressure tensor on the lattice they found gives rise to a mechanical stability that only meets the Maxwell thermodynamic condition if the pseudopotential is set to $\psi=\psi_{0} \exp \left(-\rho_{0} / \rho\right)$, where $\rho$ is the density and $\rho_{0}$ and $\psi_{0}$ are arbitrary constants. He and Doolen [8] further pointed out that the pseudopotential must be proportional to density, i.e., $\psi \propto$ $\rho$, if the continuum integration approach is applied to calculate the nonideal part of the pressure. Such treatment, which attempts to resolve the thermodynamic consistency, stimulates

*b.chen@hw.ac.uk an unrestrained accumulation of density in the liquid phase and leads to mass collapse $[8,16]$ and, subsequently, a gauge invariance in the pressure tensor [18]. Shan [19] then proposed a more general approach to address the pressure tensor on the lattice and found that the pseudopotential, expressed as $\psi=\psi_{0} \exp \left(-\rho_{0} / \rho\right)$, is thermodynamically consistent. This pseudopotential was then discovered to be associated with an increase in numerical instability and spurious velocities in practice [20].

By using Shan's approach [19], Sbragaglia and Shan [21] eventually confirmed that the SC model coincides with a free energy model when the pseudopotential is given by $\psi=$ $[\rho /(\varepsilon+\rho)]^{1 / \varepsilon}$, where $\varepsilon$ is a constant depending on the lattice. This function is self-bounded and, hence, the distribution function is prevented from accumulating excessively. It is not difficult to find that the pseudopotential $\psi=[\rho /(\varepsilon+\rho)]^{1 / \varepsilon}$ coincides with the exponential function when only nearestnode interactions are considered. This approach was later introduced to multicomponent systems by Sbragaglia and Belardinelli [22].

Numerical tests $[19,21]$ demonstrated that the exponentialbased pseudopotential could successfully simulate the liquidvapor coexistence. However, it has also been noticed that the pseudopotential lacks sufficient free parameters to represent necessary two-phase properties, such as saturation pressure, saturation densities, and speeds of sound in liquid and vapor for a lattice Boltzmann (LB) system. In other words, the simplicity of its mathematical format and the equation of state (EOS), which it presents, prevents the approach from covering a wide range of real fluids. In this regard, another approach has been proposed to simulate the real fluids by implementing practical EOSs in the LBE, whereas attempts were made to satisfy as largely as possible the Maxwell 
equal-area rule. Yuan and Schaefer [23] examined different EOSs by implementing selected EOSs directly into the SC pseudopotential model and showed that the stability and accuracy of simulations were significantly affected. Assuming the pseudopotential as $\psi \propto \rho$, Zhang and Tian [24] set the EOS of the LBE via an equilibrium distribution function; however, the approach created larger spurious velocities in comparison with the original SC model and does not guarantee the Galilean invariance [25]. Sbragaglia et al. [18] introduced a multirange pseudopotential approach, which, besides the SC forcing term, employs a second forcing term acting on the node of interest in the lattice with the aid of information from the next-nearest neighbors. The approach and its sequels [26-30] are successful in increasing numerical stability and tuning surface tension independent of density ratio and can be considered as an improved scheme of an inconsistent pseudopotential LB framework. Kupershtokh et al. [9] proposed a model that combines two approaches: local approximation and mean-value approximation; the effect of each part is determined by trial and error to remove the thermodynamic inconsistency. Huang et al. [31] used the force calculation scheme of Sbragaglia et al. [18] and, by trial and error analyses, found coefficients of the interaction force that result in an appropriate representation of the system. Colosqui et al. [32] changed the spinodal region of the EOS to eliminate the numerical error due to discretization on the lattice, for which, however, an iterative feedback loop (self-tuning procedure) has to be adopted during the simulations. Li et al. $[25,33]$ proposed a new forcing scheme in addition to recovering correct hydrodynamics, which improved the numerical stability; however, the thermodynamic inconsistency has not been completely removed, and for each individual case, the mechanical stability solution must be fitted with the solution given by the Maxwell equal-area rule.

In this study, we propose a forcing scheme for the LB pseudopotential model that is fundamentally consistent with thermodynamics and has a greater flexibility in setting a desired thermodynamic state. We introduce the proposed scheme in Sec. II with investigations of its thermodynamic consistency. In Sec. III, we demonstrate the applicability of the scheme in representing thermodynamic states of interest through simulations of planar interfaces and buoyancy-free droplets of two real fluids. Additionally, it is posited in this section that the proposed methodology provides the potential to improve the LBE for modeling multiphase flows by mitigating the unphysical side effects, such as spurious velocities. We also discuss the possible reasons behind the common problems of collapsing simulations in practical applications when an EOS of a real fluid is implemented directly into the SC model, which is discussed here. Finally, we discuss a straightforward procedure in constructing a specific two-phase lattice Boltzmann method (LBM) system with provided properties, such as pressure, densities, and sound speeds in the vapor and liquid phases.

\section{METHODOLOGY}

In the LBE model, recurring streaming and collision processes of a set of finite distribution functions $f_{i}(\mathbf{x}, t)$ reproduce the hydrodynamic behavior,

$$
f_{i}\left(\mathbf{x}+\mathbf{e}_{i} \Delta t, t+\Delta t\right)-f_{i}(\mathbf{x}, t)=-\frac{1}{\tau}\left[f_{i}(\mathbf{x}, t)-f_{i}^{\mathrm{eq}}(\mathbf{x}, t)\right],
$$

where $\mathbf{x}$ is the position, $t$ is the time, $\tau$ is the nondimensional relaxation time, $\left\{\mathbf{e}_{i}: i=0, \ldots, q-1\right\}$ are the discrete microscopic velocity vectors to the $q-1$ neighboring nodes, and $f_{i}^{\text {eq }}$ is the local equilibrium distribution function obtained from the Chapman-Enskog expansion of the Maxwellian to the second order at constant temperature [34,35]. For a two-phase system near its equilibrium state, the momentum flux tensor can be derived by employing the equilibrium distribution function and considering its isotropy on the lattice,

$$
\sum_{i=0}^{q-1} e_{i}^{\alpha} e_{i}^{\beta} f_{i} \approx \sum_{i=0}^{q-1} e_{i}^{\alpha} e_{i}^{\beta} f_{i}^{\mathrm{eq}}=\rho \theta \delta_{\alpha \beta}+\rho u_{\alpha} u_{\beta},
$$

where $\alpha$ and $\beta$ are Cartesian coordinates, $\theta$ is the lattice temperature, and $u$ is the velocity. The lattice temperature depends on the underlying lattice structure. For the case of the D2Q9 lattice, the discrete velocities are $\mathbf{e}_{0}=\mathbf{0}, \mathbf{e}_{1}=$ $(\varsigma, 0), \mathbf{e}_{2}=(0, \varsigma), \mathbf{e}_{3}=(-\varsigma, 0), \mathbf{e}_{4}=(0,-\varsigma), \mathbf{e}_{5}=(\varsigma, \varsigma)$, $\mathbf{e}_{6}=(-\varsigma, \varsigma), \mathbf{e}_{7}=(-\varsigma,-\varsigma)$, and $\mathbf{e}_{8}=(\varsigma,-\varsigma)$, where $\varsigma=$ $\Delta x / \Delta t$ is the microvelocity and $\Delta x$ is the lattice spacing. This choice of lattice sets the lattice temperature at

$$
\theta=\varsigma^{2} / 3 \text {. }
$$

The first term on the right-hand side of (2) demonstrates the well-known ideal gas pressure. To develop the approach to model nonideal fluids and two-phase systems, Shan and Chen [2] introduced a lattice force into the LBE,

$$
\mathbf{F}=-G \psi(\mathbf{x}, t) \sum_{i=1}^{N} w\left(\left|\mathbf{c}_{i}\right|^{2}\right) \psi\left(\mathbf{x}+\mathbf{c}_{i}, t\right) \mathbf{c}_{i},
$$

to describe the interaction potentials (pseudopotentials) between the fluid particles, where $G$ is the amplitude of the force, $N$ is the number of neighboring nodes that interact with the node of interest, $\mathbf{c}_{i}$ is the vector linking to those neighbors, and $w$ is the weight function that makes the interaction force dependent on the distance. The distance to the nearest neighbor is $c$, whose magnitude is equal to $\varsigma$ if we set $\Delta t=1$. It should be noted that, from a numerical point of view, the force acts as the finite difference calculation of $-\psi \nabla \psi$ on the lattice. Having more participating nodes in this calculation, the degree of isotropy of the finite difference gradient operator can be enhanced systematically, which favors reducing spurious velocities generated close to the curved interfaces $[12,18]$. Therefore, $\mathbf{e}_{i}$ vectors should be distinguished from $\mathbf{c}_{i}$ vectors because although distribution functions are streaming with velocity vector $\mathbf{e}_{i}$ to the nearest-neighbor nodes, $\mathbf{c}_{i}$ is the cutoff radius of the force that can be extended beyond the nearest neighbors.

The pseudopotential $\psi$ can be determined by satisfying the thermodynamic requirements of the system. Matching the mechanical stability along a planar interface to the Maxwell equal-area rule for a thermodynamic system in equilibrium $\int_{\rho_{v}}^{\rho_{l}}\left(p_{0}-p\right) d \rho / \rho^{2}=0$, where $\rho_{l}$ and $\rho_{v}$ are the densities of saturated liquid and vapor at a given temperature, respectively 
[19], the pseudopotential can be defined by

$$
\frac{d \psi}{\psi^{1+\varepsilon}}=\lambda \frac{d \rho}{\rho^{2}},
$$

where $\lambda$ is an arbitrary coefficient. A general solution to this partial differential equation is

$$
\psi(\rho)=\left(\frac{\rho}{\lambda \varepsilon+C \rho}\right)^{1 / \varepsilon},
$$

where $C$ is a constant and $\varepsilon=\left(6 e_{4}-2\right) /\left(6 e_{4}+1\right)$ in which $e_{4}$ is the constant obtained from the fourth-order isotropy of the force on the lattice [19] and, as shown in Sec. II B, can affect the interface properties,

$$
e_{4}=\frac{w(1)}{2}+2 w(2)+8 w(4)+25 w(5)+32 w(8)+\cdots
$$

For example, in the case of nearest-node interactions $w\left(\left|\mathbf{c}_{i}\right|^{2}\right)=0$ for $\left|\mathbf{c}_{i}\right|^{2}>2$ and from the fourth-order isotropy of the force on the lattice $w(1)=1 / 3, w(2)=1 / 12$, and $e_{4}=1 / 3$. Weights for $\left|\mathbf{c}_{i}\right|^{2}>2$ come into effect when a higher order of isotropy is needed (for more details refer to Refs. [12,18]).

\section{A. Multipseudopotential interaction scheme}

Considering the unique EOS $p=\rho \theta+G c^{2} \psi^{2} / 2$ presented by consistent pseudopotential function (6), the system is a faithful model of the liquid-vapor coexistence. However, such a consistent system is unable to present experimental data and the practical EOSs, such as those of van der Waals (VW) and Carnahan-Starling (CS). This shortcoming comes, in mathematics, from the fact that only three free parameters $-G$, $C$, and $\lambda$-are not sufficient to describe the desired relations among the properties of real fluids in two-phase coexistence, such as pressure, liquid density, vapor density, and sound speeds in the vapor and liquid phases. To our understanding, this pseudopotential represents one part of the molecular potential, and such a system lacks contributions of other parts of interparticle interactions of fluids, and hence, it is unable to represent a wide variety of experimental data of the states of real fluids.

To overcome the above mentioned shortcoming, here we introduce the concept of the multipseudopotential interparticle interaction (MPI), in comparison with the single pseudopotential interaction (SPI), as the pair interactions scheme to describe the hydrodynamic properties of real fluids, under the condition that each potential satisfies the thermodynamic requirement (5),

$$
\begin{aligned}
\mathbf{F}^{\text {total }} & =\mathbf{F}^{(1)}+\mathbf{F}^{(2)}+\cdots+\mathbf{F}^{(n)} \\
& =\sum_{j=1}^{n}-G_{j} \psi_{j}(\mathbf{x}, t) \sum_{i=1}^{N} w\left(\left|\mathbf{c}_{i}\right|^{2}\right) \psi_{j}\left(\mathbf{x}+\mathbf{c}_{i}, t\right) \mathbf{c}_{i},
\end{aligned}
$$

where $n$ is the number of pseudopotentials, $G_{j}$ is the amplitude, and $\psi_{j}$ is the consistent pseudopotential of the $j$ th part of the force,

$$
\psi_{j}(\rho)=\left(\frac{\rho}{\lambda_{j} \varepsilon+C_{j} \rho}\right)^{1 / \varepsilon} .
$$

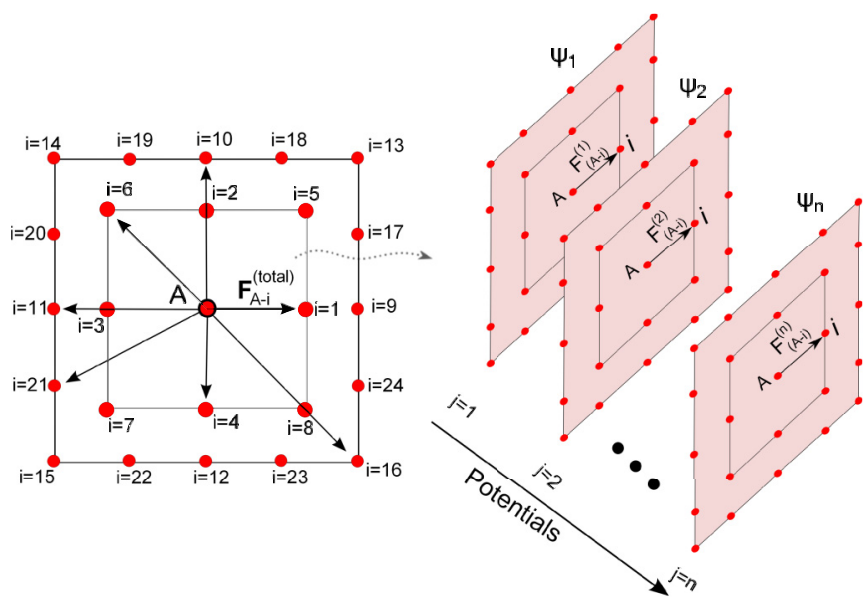

FIG. 1. (Color online) Node $A$ and the 8 nearest-neighbor nodes $(i=1,2, \ldots, 8)$ and 16 next-nearest nodes $(i=9,10, \ldots, 24)$ that it can interact with on the D2Q9 lattice. The interaction of node $A$ with each neighbor node $\mathbf{F}_{A-i}^{\text {total }}$ is composed of a series of consistent subforces. Each one represents a part of the interparticle potential.

Here, the interparticle potential is presented with the contributions of different parts. Therefore, as shown in Fig. 1, for example, the interaction force between node $A$ and its neighbors $(i=1,2, \ldots, 24)$ in a given lattice $\mathbf{F}_{A-i}^{\text {total }}$ is composed of a set of subforces at various potentials, which are the functions of particle densities, i.e., the inverse of the mean free path of particles.

It is noteworthy that the concept and physics of multipseudopotential interaction are different from those of the multirange interaction (or pseudopotential with midrange interactions) model proposed by Sbragaglia et al. [18] in which different sets of forces are introduced to the scheme with different ranges (or cutoff radii) linking to the neighborhood nodes. For instance, one set of forces with amplitude $G_{1}$ acts on the nearest nodes, and the others with amplitude $G_{2}$ act on next-nearest nodes. Such a multirange interaction model provides capabilities for the pseudopotential models, such as flexibility of setting surface tension independent of the EOS [18], achieving stable spraylike fluid [27,29], and increasing numerical stability [26,28]. However, it has to be noted that the multirange interaction scheme [18] does not take the thermodynamic consistency into consideration as all of its ranges utilize the same inconsistent pseudopotential $\psi(\rho)=\sqrt{\rho_{0}}\left[1-\exp \left(-\rho / \rho_{0}\right)\right]$, where $\rho_{0}$ is a constant.

As defined in (8) and (9) and discussed in Sec. II B, the MPI scheme we proposed is fully thermodynamically consistent. Meanwhile, in principle, the MPI scheme utilizes different pseudopotentials but requires the force interactions within a specific range and order of isotropy. For example, if the nearest-neighbor interactions are considered, all subforces only act on nearest nodes $\left\{\mathbf{F}_{A-i}^{j}: i=1,2, \ldots, 8\right\}$ and have the fourth order of isotropy. Once the interactions extend to next-nearest neighbors, all subforces act within these lattice nodes $\left\{\mathbf{F}_{A-i}^{j}: i=1,2, \ldots, 24\right\}$ and have the eighth order of isotropy (see Fig. 1). Therefore, the method is grounded on Shan's theory of calculation of the exact pressure tensor 
in pseudopotential LB systems [19] and its extension by Sbragaglia and Shan [21].

\section{B. Thermodynamic consistency analysis}

Here we prove that MPI inherently satisfies thermodynamic consistency and begin with the procedure similar to that stated in Refs. [3,19]. By employing the general rule of the force balance $\sum \mathbf{P} \cdot \mathbf{A}=\sum_{x} \mathbf{F}$ [19], the pressure tensor can be exactly expressed by the MPI force on a discrete lattice for the nearest and next-nearest interactions. Taking the D2Q9 lattice, following Ref. [19], and considering the continuum limit where all gradients are in the $x$ direction, the contribution of the total potentials of the MPI force to the pressure tensor can be calculated in the $x-y$ plane,

$$
\begin{aligned}
& P_{x x}=\sum_{j=1}^{n} \frac{G_{j} c^{2}}{2} \psi_{j}^{2}+\frac{G_{j} c^{4}}{12}\left[a_{1}\left(\frac{d \psi_{j}}{d x}\right)^{2}+a_{2} \psi_{j} \frac{d^{2} \psi_{j}}{d x^{2}}\right] \\
& P_{y y}=\sum_{j=1}^{n} \frac{G_{j} c^{2}}{2} \psi_{j}^{2}+\frac{G_{j} c^{4}}{4}\left[a_{3}\left(\frac{d \psi_{j}}{d x}\right)^{2}+a_{4} \psi_{j} \frac{d^{2} \psi_{j}}{d x^{2}}\right] \\
& P_{x y}=P_{y x}=0
\end{aligned}
$$

where $a_{1}=1-3 e_{4}, a_{2}=1+6 e_{4}, a_{3}=-4[w(5)+4 w(8)]$, and $a_{4}=a_{3}+e_{4}$.

The mechanical stability along a planar interface states that the normal component of the pressure tensor is equal to a constant pressure $p_{0}$. Hence, by adding the ideal-gas part of the pressure, we find

$p_{0}=\rho \theta+\sum_{j=1}^{n}\left\{\frac{G_{j} c^{2}}{2} \psi_{j}^{2}+\frac{G_{j} c^{4}}{12}\left[a_{1}\left(\frac{d \psi_{j}}{d x}\right)^{2}+a_{2} \psi_{j} \frac{d^{2} \psi_{j}}{d x^{2}}\right]\right\}$.

This equation, unsurprisingly, is the extended version of the pressure for the single pseudopotential force model [19] with the potentials up to the $n$th part. It is noteworthy that $e_{2}$, which is the constant of the second-order isotropy and must appear in front of the $\psi_{j}^{2}$ terms, is merged with $G_{j}$ at a more general format. Equation (13) can then be rearranged in the format including only derivatives with respect to density,

$$
\sum_{j=1}^{n} \frac{G_{j} c^{4} \psi_{j}^{1+\varepsilon}}{8(1-\varepsilon) \psi^{\prime}{ }_{j}} \frac{d}{d \rho}\left[\left(\partial_{x} \rho\right)^{2} \frac{\psi_{j}^{\prime 2}}{\psi_{j}^{\varepsilon}}\right]=p_{0}-\rho \theta-\sum_{j=1}^{n} \frac{G_{j} c^{2}}{2} \psi_{j}^{2},
$$

where $\psi^{\prime}=d \psi / d \rho$. Replacing $\psi_{j}^{1+\varepsilon} / \psi_{j}^{\prime}$ with $\rho^{2} / \lambda_{j}$, we are able to integrate all terms of (14) along the interface,

$\left(\partial_{x} \rho\right)^{2} \sum_{j=1}^{n} \frac{G_{j} c^{4} \psi_{j}^{\prime 2}}{8(1-\varepsilon) \lambda_{j} \psi_{j}^{\varepsilon}}=\int\left(p_{0}-\rho \theta-\sum_{j=1}^{n} \frac{G_{j} c^{2}}{2} \psi_{j}^{2}\right) \frac{d \rho}{\rho^{2}}$.

We can then solve (15) to find the density profile $\partial_{x} \rho$ across the interface under the boundary conditions of $\rho(-\infty)=\rho_{v}$, $\rho(+\infty)=\rho_{l}$, and $p_{0}=p\left(\rho_{l}\right)=p\left(\rho_{v}\right)$. We further utilize the fact that in both liquid and vapor phases far from the interface, density has a uniform profile $\partial_{x} \rho$, which makes all terms on the left-hand side of (15) zero; therefore, we obtain, in bulk liquid and vapor,

$$
\int_{\rho_{v}}^{\rho_{l}}\left(p_{0}-\rho \theta-\sum_{j=1}^{n} \frac{G_{j} c^{2}}{2} \psi_{j}^{2}\right) \frac{d \rho}{\rho^{2}}=0 .
$$

It is obvious that (16) is the expression of the Maxwell construction, which means, with the first assumption that each individual force is thermodynamically consistent, the summation of these interparticle forces, therefore, is thermodynamically consistent. The corresponding EOS is

$$
p=\rho \theta+\sum_{j=1}^{n} \frac{G_{j} c^{2}}{2} \psi_{j}^{2} .
$$

This virial-like EOS (17) is the result of the multipseudopotential interaction forces (8). The MPI pseudopotential proposed in this study can be further applied to predict the interfacial forces in terms of the surface tension. In the case of a planar interface, surface tension can be calculated from

$$
\sigma=\int_{-\infty}^{+\infty}\left(p_{N}-p_{T}\right) d x=\int_{-\infty}^{+\infty}\left(P_{x x}-P_{y y}\right) d x
$$

where $p_{N}$ is the local pressure normal to the interface and $p_{T}$ is the local pressure tangential to the interface. Solving Eq. (18) with the condition $\partial_{x} \rho=0$ again in both liquid and vapor phases far from the interface, we can obtain

$$
\sigma=-\frac{c^{4} e_{4}}{2} \int_{\rho_{v}}^{\rho_{l}}\left(\sum_{j=1}^{n} G_{j} \psi_{j}^{\prime 2}\right)\left(\partial_{x} \rho\right) d \rho,
$$

where $\partial_{x} \rho$ is solved by (15).

Equations (17) and (19) are the general expressions of the EOS and the surface tension for the MPI LBE model, which are thermodynamically consistent. In physics, each potential of the MPI forces provides a certain order of interparticle interactions to correct the ideal gas pressure, i.e., the first term of (17). In mathematics, each term has a set of free parameters to describe the detailed dynamics of physics. In addition, following the work of Sbragaglia and Shan [21], it can be straightforwardly demonstrated that the proposed MPI has the format that leads to a consistency between the lattice pressure tensor and the free energy density developed from the square gradient theory (see Appendix A).

\section{RESULTS AND DISCUSSION}

Herein, we practically analyze the MPI for two-phase flows by considering a buoyancy-free steady droplet in equilibrium with its vapor and liquid-vapor coexistence with a planar interface, which is the classical case studied previously for testing the interfacial models [12,23,31]. The MPI capability in reproducing the VW and CS EOS is thoroughly investigated in comparison with another common scheme. Analysis is performed regarding the recovery of the real fluid EOS, mechanical stability, thermodynamic consistency, and eventually the spurious velocities. Additionally, a straightforward procedure is proposed to preset MPI parameters to achieve the desired two-phase properties. 


\section{A. Equations of state used in simulations}

The fluids are VW and CS fluids. For the VW, the EOS,

$$
p=\frac{\rho T}{1-b \rho}-a \rho^{2}
$$

exists as the basis of many other EOSs of real fluids. Here its parameters are set as $a=9 / 49$ and $b=2 / 21$. The fluid's critical point (critical density $\rho_{c}=1 / 3 b$, critical temperature $T_{c}=8 a / 27 b$, and critical pressure $p_{c}=a / 27 b^{2}$ ) is deemed to be the reference point for nondimensionalization. The CS EOS,

$$
p=\rho T \frac{1+\eta+\eta^{2}-\eta^{3}}{(1-\eta)^{3}}-a \rho^{2},
$$

which modifies the hard sphere repulsion part of the VW equation, is a popular EOS in the pseudopotential LB community. Here $\eta=b \rho / 4, b=4$ and for $a$ two values are considered $a=1,5.5$. The properties of the critical point are $T_{c}=0.18727 a /(0.4963 b)$ and $p_{c}=0.18727 T_{c} / b$.

Weight functions are $w(1)=1 / 3$ and $w(2)=1 / 12$. The nearest-node (nodes $1-8$ in Fig. 1) interactions are employed such that, according to (7), $e_{4}=1 / 3$ and $\varepsilon$ approaches zero; therefore, if we set $C=1$, from (9), we find

$$
\psi_{j}^{M}(\rho)=\exp \left(-\lambda_{j} / \rho\right)
$$

where the superscript " $M$ " indicates the pseudopotential of the MPI. The choice of $C$ does not change the shape of the pseudopotential function of (22). Therefore, the EOS of such a system is

$$
p=\rho \theta+\sum_{j=1}^{n} \frac{G_{j} c^{2}}{2} \exp \left(-2 \lambda_{j} / \rho\right) .
$$

\section{B. Single pseudopotential and multipseudopotential interactions}

Application of merely one forcing term-herein we name it the SPI force scheme-is a common method of recovering an EOS [9,23,25,31]. The SPI scheme is used as the reference for discussions. Following Ref. [23], the single pseudopotential $\psi^{S}$ in the SC model can be obtained by the EOS directly,

$$
\psi^{S}=\sqrt{\frac{2\left[p(\rho, T)-\rho \varsigma^{2} / 3\right]}{G c^{2}}},
$$

where $p(\rho, T)$ can be calculated by the VW or CS EOS. This selection of pseudopotential makes the magnitude of $G$ nonfunctional as it will be canceled out in the calculation of the interaction force (4), although its sign can help to keep the radicand positive. This scheme makes it possible to impose the desired EOS directly on the interaction force and attempts to remove the ideal gas pressure created by streaming and collision of distribution functions. It has been demonstrated that, however, no practical EOSs placed into (24) could satisfy the thermodynamic condition (5). Moreover, in this SPI LBE scheme [23], the microvelocity is usually set to unity $\varsigma=1$ (i.e., $c=1$ ), and temperature is independently set to model different isothermal systems. As we show in the following discussion, the thermodynamic equilibrium state of the SPI will change with the value of $c$.
For the case of the SPI, the mechanical stability is characterized by the exact pressure tensor on the lattice, which is obtained from nearest-node interactions. It leads to

$$
\int_{\rho_{v}}^{\rho_{l}}\left[p_{0}-\rho \frac{\varsigma^{2}}{3}-\frac{G c^{2}}{2}\left(\psi^{S}\right)^{2}\right] \frac{\left(\psi^{s}\right)^{\prime}}{\psi^{s}} d \rho=0 .
$$

The thermodynamic consistency in this scheme is characterized by means of the Maxwell construction,

$$
\int_{\rho_{v}}^{\rho_{l}}\left[p_{0}-p(\rho, T)\right] \frac{d \rho}{\rho^{2}}=0,
$$

which is considered the benchmark.

For the case of the MPI, the mechanical stability and Maxwell thermodynamic condition are the same and identified as

$$
\int_{\rho_{v}}^{\rho_{l}}\left[p_{0}-\rho \frac{\varsigma^{2}}{3}-\sum_{j=1}^{n} \frac{G_{j} c^{2}}{2}\left(\psi_{j}^{M}\right)^{2}\right] \frac{d \rho}{\rho^{2}}=0 .
$$

Equations (25)-(27) are solved numerically along with the conditions $p_{0}=p\left(\rho_{l}\right)=p\left(\rho_{v}\right)$ to find $\rho_{l}, \rho_{v}$, and $p_{0}$ for each scheme. Examining (27), recalling (23) for $p_{0}$, and knowing $\varsigma \propto c$, it can be realized that $c$ can be crossed out from (27). It means that the MPI scheme under grid refinement or scalability, i.e., change in $c$, shows the same densities in the given equilibrium state. Nonetheless, the mechanical stability of the SPI Eq. (25) is affected by the choice of $c$ as $\left(\psi^{s}\right)^{\prime} / \psi^{s}$ is a nonlinear function of $c$.

\section{Simulation setup}

All simulations are run on a two-dimensional square lattice including nine velocities (D2Q9). The computational domain contains $200 \times 200$ nodes. The nondimensional relaxation time is set to unity $\tau=1$. The internal forces are embedded in the LBE by the method proposed by Guo et al. [36], which reproduces Navier-Stokes equations to the second order. For the case of the planar interface, half of the domain is filled with liquid density and the other half with the vapor density. For the case of the curved interface, a droplet with the radius $r_{0}=40$ lattice unit is placed initially in the middle of the domain. The periodic condition is applied at all boundaries. $\rho_{l}$ and $\rho_{v}$ are initially set, in a sense, to ensure that the system is in the saturation state. To avoid initial instability, a diffuse interface should be adopted at the beginning of the simulations. We utilize the suggestion of Ref. [31],

$$
\rho(r)=\frac{\rho_{l}+\rho_{v}}{2}-\frac{\rho_{l}-\rho_{v}}{2} \tanh \left(\frac{r-R}{W}\right),
$$

where $W$ controls the interface width and, in all simulations, is set at $W=5, R$ is the distance between the interface and the reference point, and $r$ is the variable distance from the reference point. For the case of the circular drop, the reference point is the center of the drop, and for the case of the planar interface, it can be one of the boundaries parallel to the phase interface. The maximum spurious velocity $|\mathbf{u}|_{\max }$, which is a common parameter to assess the LBM force schemes [23,31], is measured regularly during the runs of drop simulations. The error of the simulations are calculated from deviation from 


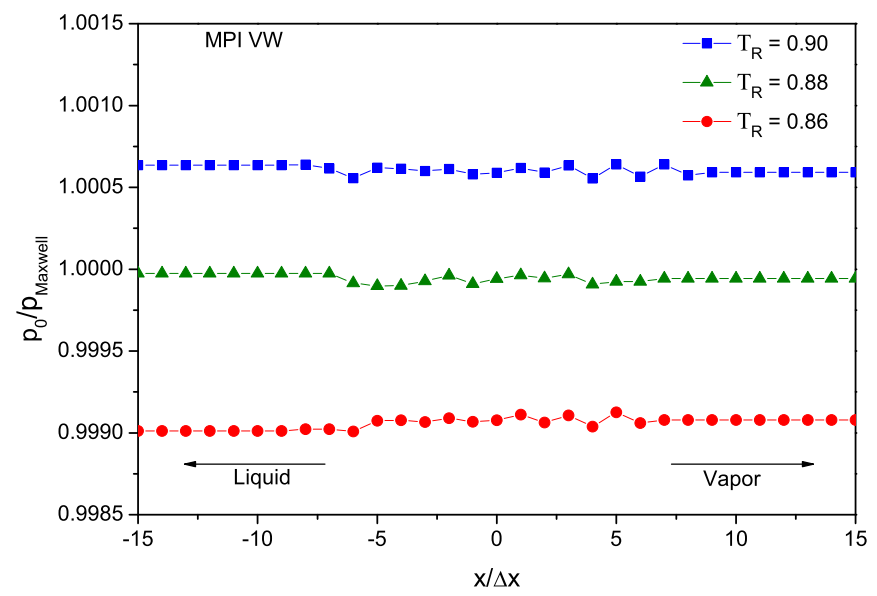

FIG. 2. (Color online) Profile of the total normal component of pressure tensor (normalized by $p_{\text {Maxwell }}$ ) along the phase interface for the MPI scheme showing the VW EOS at different temperatures.

Maxwell construction results (26),

$$
\text { Error }=\frac{\left|\rho_{\text {simulation }}-\rho_{\text {Maxwell }}\right|}{\rho_{\text {Maxwell }}} .
$$

As the MPI EOS is scalable, as an option, we can simply set the lattice temperature as the scale of a simulation and, therefore, EOS temperature $T$ coincides with lattice temperature $\theta$. Such a choice fixes the microvelocity at $\varsigma=\sqrt{3 T}$, which is the strategy we selected in the case of the VW fluid. In order to recover the VW fluid, we fit the MPI EOS onto the VW EOS, whereby, by trial and error, we found that four potentials in (23) with their free parameters $G_{j}$ and $\lambda_{j}$ are needed. For the case of the CS fluid, we impose no constraint on $\varsigma$ and disconnect it from the system temperature as in the SPI scheme. We employ it only as another free parameter for fitting. Following this strategy, by trial and error, we found only $n=3$ pseudopotentials in (23) are needed to implement the $\mathrm{CS}$ into the MPI. The details of the curve fittings are provided in Appendix B.

\section{Flat phase interface}

The first set of simulations is run to build planar interfaces using the MPI scheme and the VW EOS. As spelled out in Sec. III B, according to the mechanical stability of a flat interface, the total normal component of the pressure tensor $p_{0}=P_{x x}+\rho \theta$ across the phase interface must be constant. Here, we can compute $P_{x x}$ from Eq. (10) where $a_{1}=0$ and $a_{2}=3$. The second spatial derivative of the pseudopotential is obtained by use of the second-order central difference approximation. Figure 2 shows $p_{0}$ nondimensionalized by $p_{\text {Maxwell }}$ along the phase interface for $T_{R}=0.86,0.88,0.90$. Calculating the change in pressure along the interface $\Delta p=$ $\left|p_{0}-p_{\text {liquid }}\right| / p_{\text {liquid }}$, we estimated the maximum error of $0.011 \%, 0.008 \%$, and $0.008 \%$ for each case, respectively, which validates the mechanical stability of the system. The errors are suggested to associate with higher-order terms of the pressure tensor, which are neglected in Eq. (10).

The second set of simulations is run to investigate the MPI using the CS EOS when constructing a planar interface
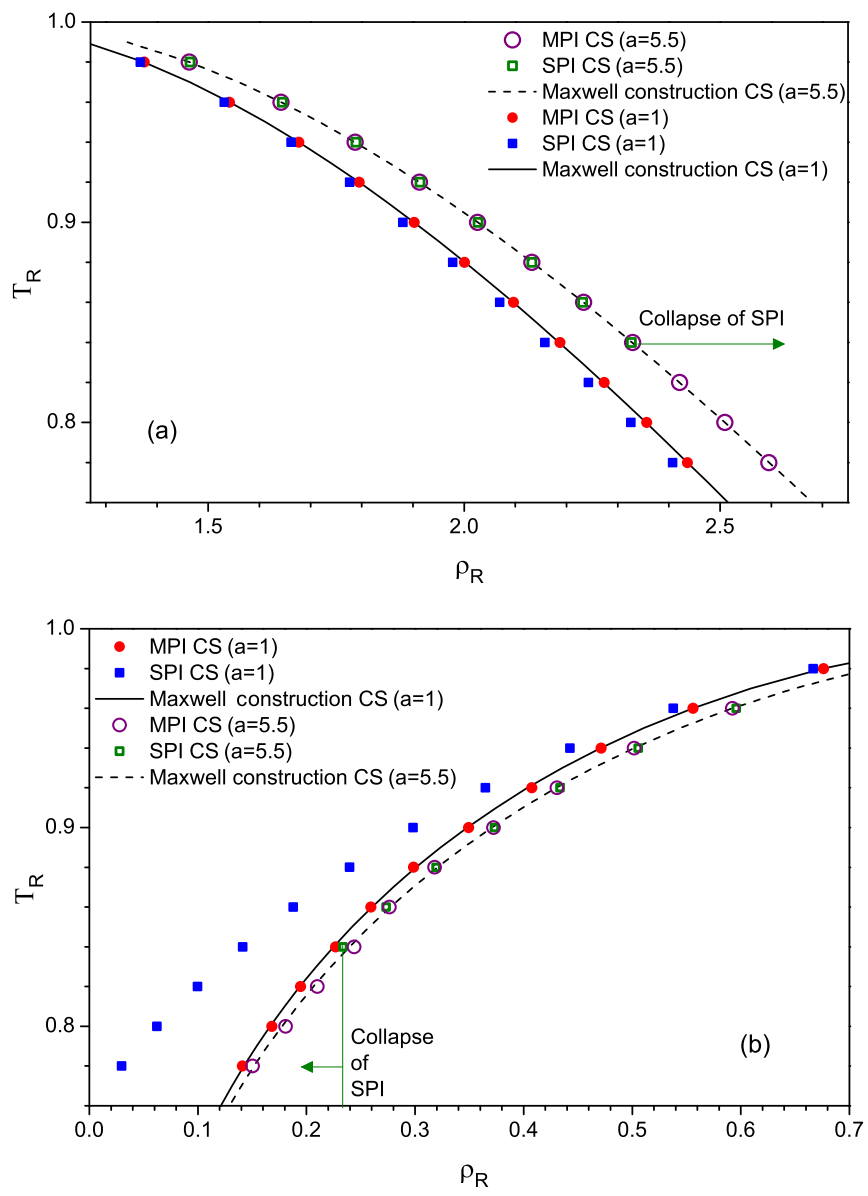

FIG. 3. (Color online) Results obtained from the MPI and SPI simulations of the CS EOS in comparison with analytical results of the Maxwell construction: (a) reduced density of liquid versus reduced temperature and (b) reduced density of vapor versus reduced temperature. The thin lines indicate where the SPI simulations collapse.

in comparison with the SPI. Figure 3(a) demonstrates the equilibrium (reduced) density versus (reduced) temperature achieved for the liquid branch of the liquid-vapor coexistence curve. For the case of $a=1$, the SPI starts deviating from the Maxwell construction as temperature decreases. At $T_{R}=0.78$, the error is $1.2 \%$. On the other hand, the MPI results follow those of the liquid branch very well where the error at $T_{R}=$ 0.78 is $0.3 \%$. If the attraction part of the CS EOS increases to $a=5.5$, both the SPI and the MPI show satisfactory results, although the SPI simulations unconditionally collapse if the temperature decreases to less than $T_{R}=0.84$. This is not the case for the MPI, which successfully simulates the Maxwell construction at the lower temperature $T_{R}=0.78$. As the case of the CS EOS with parameters of $a=1$ and $b=4$ has been used commonly in the literature, we deliberately have chosen $a=5.5$ and $b=4$ to show a shortcoming of the SPI schemes, which will be discussed in Sec. IIIF. The vapor branch of the coexistence curve of the CS EOS is shown in Fig. 3(b). In the case of $a=1$, the deviation of the SPI from the Maxwell construction is more severe than the liquid branch where the error reaches $79 \%$ at $T_{R}=0.78$. In contrast, the MPI error at that temperature is about $1 \%$. For the case of $a=5.5$, the SPI 

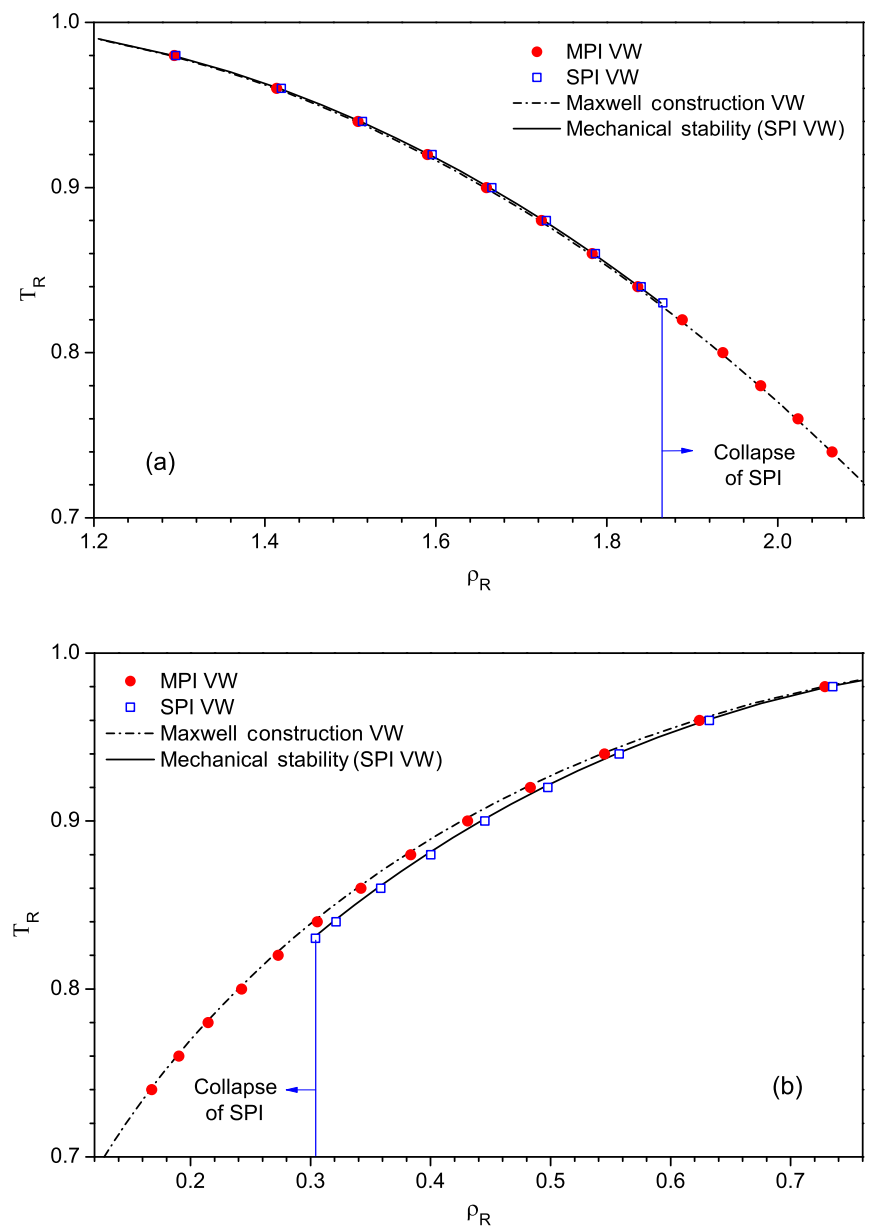

FIG. 4. (Color online) Results obtained from the MPI and SPI simulations of the VW EOS in comparison with analytical results of the Maxwell construction and mechanical stability: (a) reduced density of liquid versus reduced temperature and (b) reduced density of vapor versus reduced temperature. The vertical thin lines indicate where the SPI simulations collapse.

shows improvement where the error at $T_{R}=0.84$ is $2.6 \%$ and the MPI shows $1.8 \%$. Nonetheless, as described above, the SPI collapses as the temperature decreases to less than $T_{R}=0.84$.

\section{E. Circular droplet}

The third set of simulations is devoted to the case of the buoyancy-free steady VW droplet. Figure 4 shows the reduced density of the saturated liquid and vapor versus the reduced temperature. Both schemes satisfactorily predict the liquid branch of the VW EOS, shown in Fig. 4(a), whereby the maximum deviation from the Maxwell construction by the SPI scheme is $0.6 \%$ at $T_{R}=0.92$ and by the MPI scheme is $0.4 \%$ at $T_{R}=0.98$. On the vapor branch as predicted and shown in Fig. 4(b), the SPI scheme exhibits mechanical stability (25) and deviates gradually from the Maxwell construction (26) with decreasing temperature. It has been noticed that the SPI scheme works well with reduced temperatures higher than 0.83 but collapses when the reduced temperature decreases further, the same as for the CS EOS. The details on this collapse behavior, observed as the major shortcoming of the SPI scheme, will be

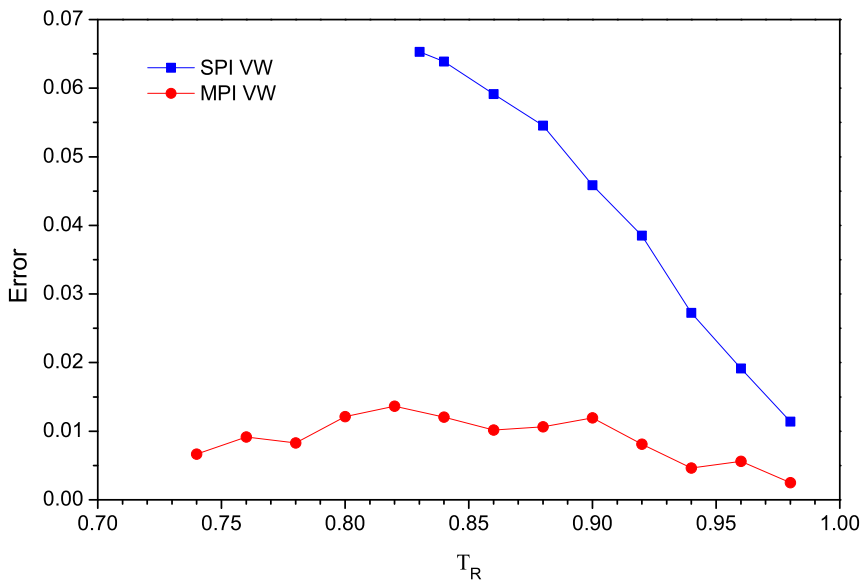

FIG. 5. (Color online) Errors due to deviation from the vapor branch of the Maxwell construction (26). Results are obtained from the MPI and SPI simulations of the VW EOS.

discussed in Sec. III F. In contrast, the MPI scheme, down to lower temperatures, demonstrates good agreement with the thermodynamic condition (26). This behavior can be seen clearly in Fig. 5, which illustrates the error resulting from the deviation from the vapor branch of the Maxwell construction (26). Whereas the SPI scheme error increases remarkably with the decrease in temperature, the errors generated by the MPI stay consistently under $1.5 \%$ and are generally independent of temperature. When the temperature decreases, in principle, the liquid-vapor density ratio rises; hence, the MPI predictions are generally steady while the density ratio increases.

The spurious velocities produced by the SPI (using the VW EOS) and the MPI (using the VW EOS) schemes are examined, and the results are shown in Table I. Spurious velocities for both models rise as the saturation temperature decreases. The maximum spurious velocities observed from the MPI simulations at different temperatures are about $50 \%$ of those obtained from the SPI. The details are demonstrated by the distribution of spurious velocities in Fig. 6 where both schemes are applied to simulate the VW fluid at $T_{R}=0.84,0.88,0.94$ and the results are depicted at the same scale. The spurious velocities generated at left, right, top, and bottom of the SPI

TABLE I. Maximum spurious velocities of the VW drop at various temperatures and their reduction levels by use of the MPI over the SPI.

\begin{tabular}{lccc}
\hline \hline$T_{R}$ & $|\mathbf{u}|_{\max }^{\mathrm{SPI}} / c$ & $|\mathbf{u}|_{\max }^{\mathrm{MPI}} / c$ & Reduction $(\%)$ \\
\hline 0.74 & & $6.85 \times 10^{-2}$ & \\
0.78 & & $2.48 \times 10^{-2}$ & \\
0.84 & $1.63 \times 10^{-2}$ & $8.40 \times 10^{-3}$ & 48.57 \\
0.86 & $1.26 \times 10^{-2}$ & $5.90 \times 10^{-3}$ & 53.02 \\
0.88 & $9.16 \times 10^{-3}$ & $4.45 \times 10^{-3}$ & 51.40 \\
0.90 & $6.32 \times 10^{-3}$ & $2.99 \times 10^{-3}$ & 52.67 \\
0.92 & $3.93 \times 10^{-3}$ & $1.85 \times 10^{-3}$ & 52.93 \\
0.94 & $2.21 \times 10^{-3}$ & $1.03 \times 10^{-3}$ & 53.50 \\
0.96 & $9.48 \times 10^{-4}$ & $4.34 \times 10^{-4}$ & 54.24 \\
0.98 & $2.19 \times 10^{-4}$ & $1.03 \times 10^{-4}$ & 52.76 \\
\hline \hline
\end{tabular}




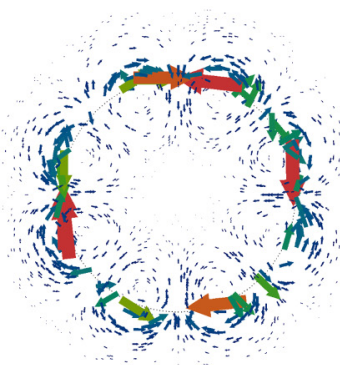

(a)

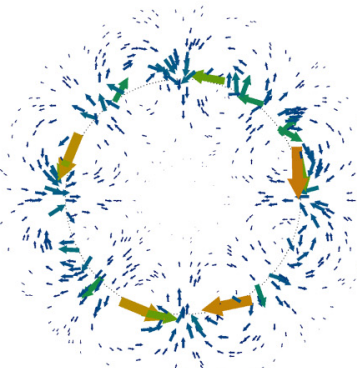

(c)

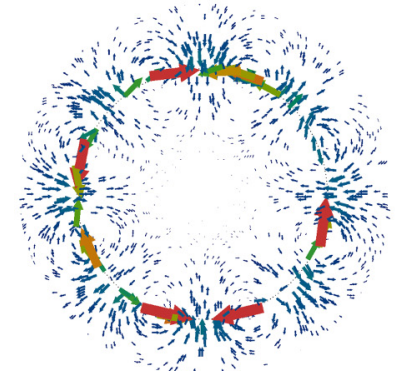

(e)

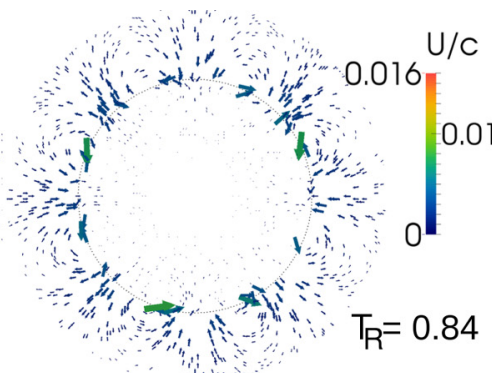

(b)

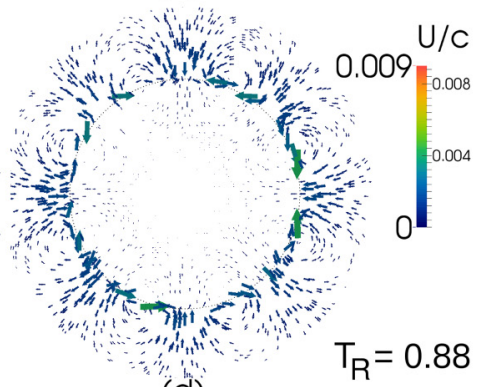

(d)

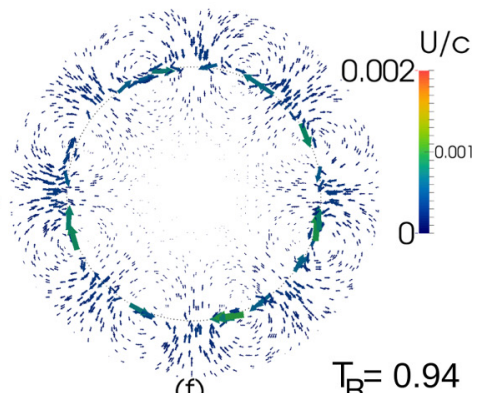

(f)
$T_{R}=0.94$
FIG. 6. (Color online) Spurious velocities are shown, the SPI: (a), (c), and (e); and the MPI: (b), (d), and (f) for simulating the VW fluid at different temperatures with droplets having reached the equilibrium state.

droplets are much greater than what are observed at the MPI droplets.

\section{F. Physical analysis of the SPI}

Running different cases with the SPI scheme, we have found that LBE simulations collapse and the mechanical stability (25) integrations tend to diverge at low temperatures (see Figs. 3 and 4) where obviously spurious velocities are not the cause. The collapse of simulations for the VW fluid has been reported by Ref. [23]; however, the reasons for its occurrence were not clarified. It is in fact due to, considering Eq. (24), the difficulty in guaranteeing $\psi^{S}$ to be a real value. For example, by setting $G<0, \psi^{S}$ could only be a real value if $p-\rho / 3 \leqslant 0$. We discuss the details by using the reduced format of the equation $p_{R}-(3 b / a) \rho_{R}$, which is depicted versus the reduced density for the VW EOS at three different temperatures in Fig. 7. It can be seen from Fig. 7 that there are regions close to the subcooled liquid and superheated vapor where the term above is positive. If only these states are simulated, it is possible to set $G$ as a positive value and make $\psi^{S}$ physically meaningful. Nonetheless, if a saturated fluid is modeled, the change in sign of $G$ may not be of any help. As shown in Fig. 7, at the high

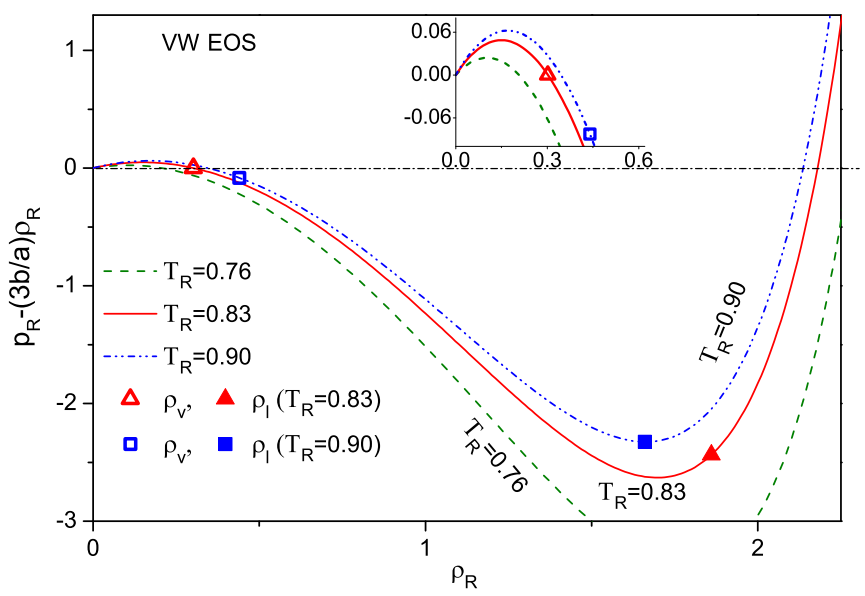

FIG. 7. (Color online) Behaviors of $p_{R}-(3 b / a) \rho_{R}$, which is used to compute $\psi^{S}$ as a function of density for the VW equation.

temperature of $T_{R}=0.90$, a physically meaningful $\psi^{S}$ can be obtained for both liquid and vapor if we set $G<0$ since the term $p_{R}-(3 b / a) \rho_{R}<0$. However, if temperature is reduced to $T_{R}<0.83\left[p_{R}-(3 b / a) \rho_{R}=0\right.$ at $\left.T_{R}=0.83\right]$, the term $p_{R}-(3 b / a) \rho_{R}$ is positive for vapor while being negative for liquid. Lower than this temperature, obviously, the SPI scheme using the VW EOS fails to calculate $\psi^{S}$ by (24) either through setting $G$ as a positive or a negative value. The threshold temperature $T_{R}=0.83$, which is identified in this study, is found by numerically solving Eq. (25) for the case of the VW fluid with parameters $a=9 / 49$ and $b=2 / 21$. Physically, this is the reason that no results could be illustrated in Figs. 3 (for case $a=5.5$ ) and 4 from the SPI model for lower temperatures. The threshold temperature depends on the ratio of $b / a$ for the VW fluids. This means that the available domain of the SPI must be investigated for a given fluid individually in order to preclude the collapse of the simulation.

It should be noted that the difficulty in guaranteeing the real value for $\psi^{S}$ is a general drawback of the SPI scheme using Eq. (24), as long as a VW-like EOS, such as the EOSs of Peng-Robinson, Redlich-Kwong, and Carnahan-Starling, is employed.

\section{G. Physical analysis of the MPI}

The LBE EOS recovers the CS EOS accurately when the proposed MPI scheme is used as shown in the inset graph of Fig. 8. The contributions of each pseudopotential to the dynamic properties of a CS fluid is examined with the aid of the potential factors,

$$
\Pi_{j}=\frac{1}{\rho T} \frac{G_{j} c^{2}}{2} \psi_{j}^{2},
$$

which are shown in Fig. 8 at the reduced temperature of $T_{R}=0.8$ versus reduced density, which can represent the inverse of the mean free path of a particle. As demonstrated in Fig. 8, when the density approaches zero, all potential factors approach zero, and the fluid acts as an ideal gas. As density increases, $\Pi_{1}$, which has the nature of attraction of particles, begins to play a relatively major role in controlling the long-distance-particles (low density) interactions in the vapor 


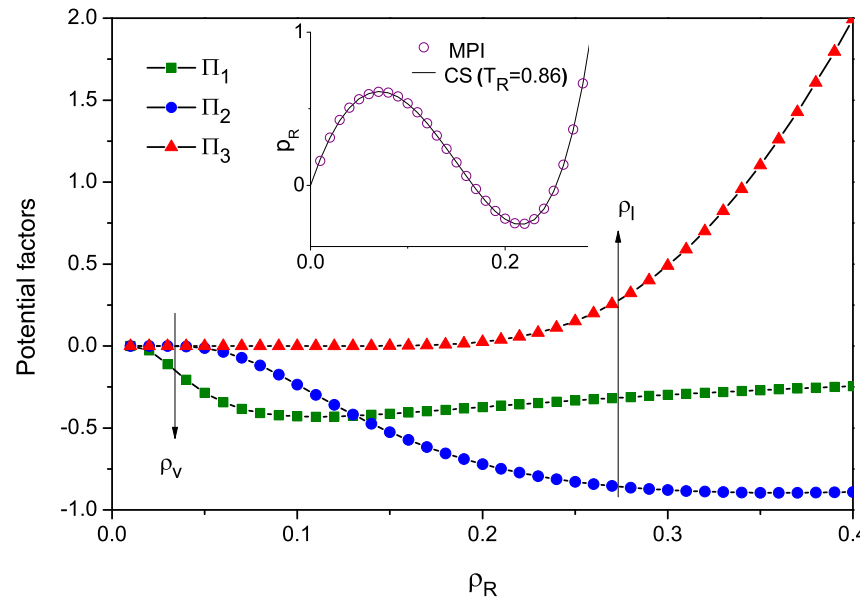

FIG. 8. (Color online) The potential factors of the MPI model contributed to reproduction of the CS isotherm at temperature $T_{R}=$ 0.86. The parameters are listed in Appendix B.

region. In the interface region of liquid and vapor, $\Pi_{2}$, which has the nature of additional attraction of particles, gradually becomes more dominant for coupling the long-distance with short-distance (high density) interactions. Finally, approaching the liquid phase, $\Pi_{2}$ becomes saturated, but the relatively powerful $\Pi_{3}$, which has a repulsion nature of particles, dominates in simulating the short-distance interactions. Whereas $\Pi_{1}$ and $\Pi_{2}$ act as the attraction moieties of the interactions, $\Pi_{3}$ reflects the hard sphere model of the CS EOS. It should be noted that $\Pi_{3}$ is still a soft force that will be saturated in a place much higher than $\Pi_{1}$ and $\Pi_{2}$. These results demonstrate two important physical characteristics of the MPI; first, the mass collapse (or overaccumulation of mass) is prevented by powerful repulsive $\Pi_{3}$, which differs from, at least, the original pseudopotential idea that the hard sphere model is achieved by the saturation of the pseudopotential. Second, considering the fact that, here, the interactions are between nearest nodes only, all the physics of molecular short-distance repulsion and long-distance attraction are achieved without the need for increasing the LB force cutoff radius (i.e., extending the interactions to the next-nearest nodes or more). This advantage comes from the nature of the MPI that the total interaction between two specific nodes is not solely attractive or repulsive. On the other hand, the physical meaning of the SPI scheme is unclear as discussed in Sec. III F. The repulsion part of the original SC model is provided by the ideal pressure created by streaming and collisions of distribution functions. This leads to the two-phase systems in which the speed of sound in liquid is less than the vapor phase and, perhaps, generates instability [9]. As shown in this paper, the MPI follows the VW and CS EOSs well, indicating that the MPI models the sound speed of two fluids appropriately. The MPI shows that in two-phase systems in which a jump of densities exists, the multiple pseudopotentials are necessary to explain the underlying physical molecular interactions in the region of two neighboring nodes on the lattice. Such an MPI makes it possible that the different forces take the responsibility for interparticle interactions in the different regions of the thermodynamic equilibrium state.
TABLE II. Thermodynamic properties of the saturated VW fluid with the parameters $a=9 / 49, b=1 / 21$ and the critical point of $p_{c}=3, \rho_{c}=7$, and $T_{c}=1.143$.

\begin{tabular}{lccccc}
\hline \hline$\frac{T}{T_{c}}$ & $p_{\text {sat }}$ & $\rho_{v}$ & $\rho_{l}$ & $(\partial p / \partial \rho)_{v}$ & $(\partial p / \partial \rho)_{l}$ \\
\hline 0.80 & 1.150 & 1.678 & 13.529 & 0.464 & 2.254 \\
0.86 & 1.594 & 2.369 & 12.457 & 0.378 & 1.362 \\
0.90 & 1.941 & 2.980 & 11.601 & 0.302 & 0.873 \\
\hline \hline
\end{tabular}

\section{H. Preset thermodynamic state}

In Secs. III E and III D, we showed that the equation of state of the MPI can be constructed by fitting on the real EOSs, such as the VW and CS, and, therefore, the LBE simulation system reflects the equilibrium states of those EOSs. Alternatively, a simple method rather than using curve fitting can be developed by implementing the thermodynamic conditions of the liquid-vapor phase equilibrium into the MPI. Similar work has been carried out by Colosqui et al. [32] where a piecewise linear EOS is adopted. Because the pseudopotential applied in their study violates thermodynamic consistency, a self-tuning procedure was adopted during the simulations to perturb the unstable branch of the EOS and satisfy the consistency. In contrast, the MPI is intrinsically consistent and capable of precisely representing the preset pressure tensor as shown in Fig. 2 without additional corrections.

Taking the VW EOS as an example, we briefly demonstrate the method of presetting the MPI as follows. The VW fluid with the parameters $a=9 / 49, b=1 / 21$ is considered, which has the critical point of $p_{c}=3, \rho_{c}=7$, and $T_{c}=1.143$. The characteristics of the saturated fluid at temperature $T$, such as saturation pressure $p_{\text {sat }}$, density of liquid $\rho_{l}$, density of vapor $\rho_{v}$, sound speed in the liquid phase $\sqrt{(\partial p / \partial \rho)_{l}}$, and sound speed in the vapor phase $\sqrt{(\partial p / \partial \rho)_{v}}$, can be determined from the EOS and employed for presetting the MPI LB system. Table II shows these values at $T_{R}=0.80,0.86,0.90$. To model one of these states in a LB system, we need to impose the five characteristics mentioned above on the MPIEOS (23) and thus identify the MPI forces.

Here we set the lattice temperature at fluid temperature $\theta=T$, i.e., $\varsigma=\sqrt{3 T}$. Therefore the first term of (23) is identified. For the sake of simplicity and precluding the nonlinearity of equations, only $G_{j}$ are considered as unknowns in (23). Therefore five pseudopotentials $n=5$ are considered. Based on the pattern of $\lambda_{j}$ in the VW and CS EOSs (see Appendix B), we can set $\lambda_{1}=m \rho_{c}, \lambda_{j}=4^{j-1} \lambda_{1}$ and then

$$
\begin{aligned}
p= & \rho T+\frac{3 T G_{1}}{2} \exp \left(-2 m \rho_{c} / \rho\right) \\
& +\sum_{j=2}^{5} \frac{3 T G_{j}}{2} \exp \left(-4^{j} m \rho_{c} / 2 \rho\right),
\end{aligned}
$$

where $m$ is a free parameter discussed in the following.

The conditions of pressure and sound speeds can constrain (31) and its first derivative. Generally, the pseudopotential models achieve a two-phase coexistence based on the VW loop observed in EOSs. Therefore, to have such a shape in (31), the Maxwell equal-area rule (27) and its boundary condition are put into effect. Variable $m$ is chosen to guarantee a VW- 


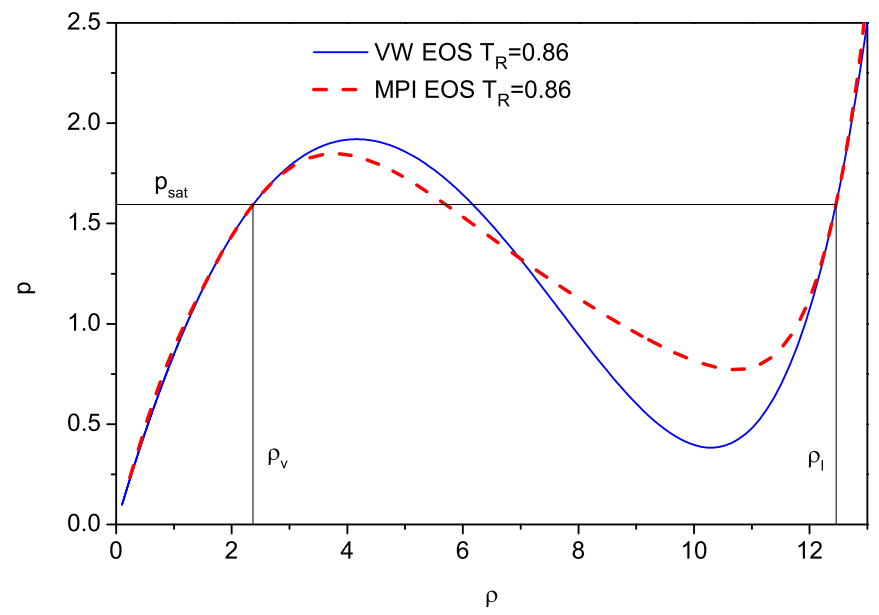

FIG. 9. (Color online) The preset EOS of the MPI versus the VW EOS at $T_{R}=0.86$. In addition to creating a VW-like loop, the MPI curve mimics the VW curve in the vicinity of the saturation densities.

like loop with a single maximum pressure point, and a single minimum pressure point can be created without singularity in the system of linear equations. Here, we set it as $m=0.057$. All five equations are derived and can be found in Appendix C for reference. Finally, a system of five linear equations can be solved instantly to find $\left\{G_{j}: j=1-5\right\}$. Then, a LBE MPI system is set up. Figure 9 shows the MPI EOS obtained via this method, in comparison with the VW EOS at $T_{R}=0.86$. As we expected a VW-like loop is achieved where the MPI curve lays on the VW EOS in the vicinity of saturation liquid density and vapor density.

We further examined the preset MPI by performing simulations for the cases of the planar interface. The simulation results are given in Fig. 10, which show the density profile along the planar interfaces. The MPI satisfactorily reproduces the input parameters. The errors due to deviation of reproduced $\rho_{v}$ and $\rho_{l}$ from the input $\rho_{v}$ and $\rho_{l}$ are $0.002 \%$ and $0.0002 \%$ for the case of $T_{R}=0.90,0.02 \%$ and $0.0009 \%$ for the case of $T_{R}=0.86$, and $0.06 \%$ and $0.001 \%$ for the case of $T_{R}=0.80$, respectively. The errors should be associated with

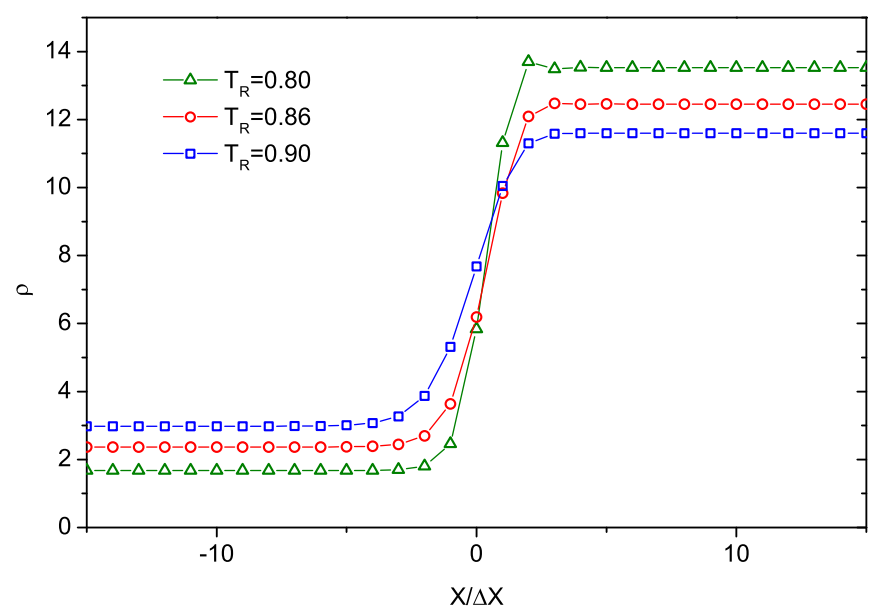

FIG. 10. (Color online) Profile of density along flat interfaces for the preset MPI at $T_{R}=0.80,0.86,0.90$. the higher-order terms of Eq. (10), which are neglected, along with spurious velocities as a consequence of lack of higher degrees of isotropy.

\section{CONCLUSION}

To summarize, we have developed a scheme based on the original SC model [2,3] to eliminate the numerical errors as a consequence of the discretization effect of the lattice. In this framework, the real EOSs and experimental data can be consistently implemented into the LB system. The fundamental ingredients of the original method are preserved, whereas the forcing scheme is further developed to include different portions of particle interactions. The outcome EOS of the MPI scheme has a virial-like shape with an arbitrary number of terms and free parameters. Each term is assigned to a pseudopotential whose effect is dependent on the mean free path of the particles.

The proposed model is compared with the commonly used SPI scheme in reproducing the VW and CS EOSs and other major characteristics of two-phase flows. It is found that, first of all, in contrast to the SPI scheme, the MPI scheme is scalable and shows the independency of equilibrium densities under grid refinement. In other words, its mechanical stability condition is not influenced by the choice of the lattice spacing. Second, when reproducing the Maxwell construction, both methods show small discrepancies at the saturated liquid branch. Regarding the vapor branch, the SPI prediction deviates significantly with a decrease in temperature, whereas the MPI scheme shows much better results, and their accuracies are independent of temperature, the density ratio, and the type of chosen EOS.

The MPI scheme used in this paper only considers the nearest-node interactions, but also the interaction between two nodes is not solely attractive or repulsive. The MPI scheme adjusts the interactions automatically according to local densities. Such a behavior can effectively reflect the underlying molecular interactions.

A shortcoming of the SPI scheme in recovering the VW-like EOSs is addressed. There exist the thermodynamic states where the SPI pseudopotential has real values in the liquid (vapor) region and imaginary values in the vapor (liquid) region, thereby causing the collapse of simulations. This problem could be resolved by constraining the parameters of simulations by decreasing the degree of freedom for engineering applications. The nature of the MPI scheme is free from this shortcoming.

An alternative procedure in simply achieving a specific MPI two-phase system is suggested using only the properties of pressure, liquid density, vapor density, and sound speeds in vapor and liquid phases. The results from the simulated system show good agreement with the input parameters.

\section{ACKNOWLEDGEMENT}

This research was supported by the FP7 Cooperation Work Programme under Grant No. 265847-FP7-OCEAN, the Natural Environment Research Council under Grant No. NE/H013970, and the Carbon Management Canada Network of Centres of Excellence, (CMC), Canada. 


\section{APPENDIX A}

The aim of this appendix is to support the discussion in Sec. II B and show that the pressure tensor of the MPI scheme in the case of one-dimensional interface Eq. (10) matches a free energy density. Following Ref. [21], we start from the free energy density,

$$
H=f(\rho)+\varphi(\rho)\left(\partial_{x} \rho\right)^{2}-l \rho,
$$

where $f$ is the bulk free energy, $l$ is a Lagrange multiplier, and $\varphi(\rho)$ is a function depending only on the density. From the equilibrium equation $\frac{\delta H}{\delta \rho}=0$, it can be shown that [21]

$$
-\rho^{2} \frac{d}{d \rho}\left[\frac{\varphi\left(\partial_{x} \rho\right)^{2}}{\rho}\right]=p_{0}-p_{b},
$$

where $p_{b}$ is the bulk pressure. Equating the left-hand sides of (14) and (A2), we find

$$
\sum_{j=1}^{n} \frac{G_{j} c^{4} \psi_{j}^{1+\varepsilon}}{8(1-\varepsilon) \psi^{\prime}{ }_{j}} \frac{d}{d \rho}\left[\left(\partial_{x} \rho\right)^{2} \frac{\psi_{j}^{\prime 2}}{\psi_{j}^{\varepsilon}}\right]=-\rho^{2} \frac{d}{d \rho}\left[\frac{\varphi\left(\partial_{x} \rho\right)^{2}}{\rho}\right] .
$$

By multiplying all terms by $d \rho / \rho^{2}$ and integrating by parts, we obtain

$$
\begin{aligned}
& \sum_{j=1}^{n} \frac{G_{j} c^{4}}{8(1-\varepsilon)}\left\{\frac{\psi_{j}^{1+\varepsilon}}{\psi^{\prime}{ }_{j} \rho^{2}}\left[\left(\partial_{x} \rho\right)^{2} \frac{\psi_{j}^{\prime 2}}{\psi_{j}^{\varepsilon}}\right]\right. \\
& \left.-\int\left[\left(\partial_{x} \rho\right)^{2} \frac{\psi_{j}^{\prime 2}}{\psi_{j}^{\varepsilon}}\right] \frac{d}{d \rho}\left[\frac{\psi_{j}^{1+\varepsilon}}{\psi^{\prime}{ }_{j} \rho^{2}}\right] d \rho\right\}=-\frac{\varphi\left(\partial_{x} \rho\right)^{2}}{\rho} .
\end{aligned}
$$

Rearranging Eq. (A4), the $\varphi(\rho)$ function can be determined as

$$
\begin{aligned}
\varphi(\rho)= & -\sum_{j=1}^{n} \frac{G_{j} c^{4}}{8(1-\varepsilon)} \\
& \times\left\{\frac{\psi_{j} \psi^{\prime}{ }_{j}}{\rho}-\frac{\rho}{\left(\partial_{x} \rho\right)^{2}} \int \frac{\left(\partial_{x} \psi_{j}\right)^{2}}{\psi_{j}^{\varepsilon}} \frac{d}{d \rho}\left[\frac{\psi_{j}^{1+\varepsilon}}{\psi^{\prime}{ }_{j} \rho^{2}}\right] d \rho\right\} .
\end{aligned}
$$

Since Eq. (A2) was derived with the assumption that $\varphi$ is a function of the density and not a function of the gradient of the density, the second term in the curly brackets should be zero. To enforce this condition, for all pseudopotentials we set

$$
\begin{aligned}
\frac{d}{d \rho}\left[\frac{\psi_{j}^{1+\varepsilon}}{\psi^{\prime}{ }_{j} \rho^{2}}\right] & =0, \\
\varphi(\rho) & =-\sum_{j=1}^{n} \frac{G_{j} c^{4}}{8(1-\varepsilon)} \frac{\psi_{j} \psi^{\prime}{ }_{j}}{\rho} \\
& =-\sum_{j=1}^{n} \frac{G_{j} c^{4}}{8 \lambda_{j}(1-\varepsilon)} \frac{\rho\left(\psi^{\prime}{ }_{j}\right)^{2}}{\psi_{j}^{\varepsilon}} .
\end{aligned}
$$

Equation (A6) exactly reproduces the thermodynamic condition (5), which demonstrates the consistency of the pressure tensor with the Maxwell equal-area rule and the free
TABLE III. Parameters at which the MPI follows the VW EOS with $a=9 / 49$ and $b=2 / 21$ for $n=4$.

\begin{tabular}{lcccccc}
\hline \hline$T_{R}$ & 0.78 & 0.82 & 0.86 & 0.90 & 0.94 & 0.98 \\
$\varsigma$ & 1.156 & 1.186 & 1.214 & 1.242 & 1.269 & 1.296 \\
$G_{1}$ & 15241 & 8478 & 5336 & 3474 & 1795 & 1721 \\
$G_{2}$ & -2.89 & -2.94 & -2.790 & -2.61 & -3.03 & -2.54 \\
$G_{3}$ & -17.2 & -15.4 & -13.7 & -12.2 & -10.8 & -9.32 \\
$G_{4} \times 10^{3}$ & -238 & -283 & -277 & -267 & -430 & -415 \\
$\lambda_{1}$ & 31.57 & 29.31 & 27.56 & 25.99 & 23.70 & 23.44 \\
$\lambda_{2}$ & 1.719 & 1.850 & 1.873 & 1.885 & 2.250 & 2.208 \\
$\lambda_{3}$ & 5.105 & 5.185 & 5.155 & 5.115 & 5.415 & 5.110 \\
$\lambda_{4} \times 10^{3}$ & 386.2 & 442.8 & 452.0 & 458.8 & 622.0 & 605.0 \\
$R^{2}$ & 1.0 & 1.0 & 1.0 & 1.0 & 1.0 & 1.0 \\
\hline \hline
\end{tabular}

energy model. In the domain, far away from density gradients, the bulk free energy is

$$
f=\rho \theta \ln \rho-\theta+\sum_{j=1}^{n} \frac{G_{j} c^{2} \rho}{2} \int_{r_{0}}^{\rho} \frac{\psi_{j}^{2}(n)}{n^{2}} d n,
$$

where $r_{0}$ is a constant. Substituting (6) into (A8) and calculating the integration, the bulk free energy simplifies to

$$
f=\rho \theta \ln \rho-\theta+\sum_{j=1}^{n} \frac{G_{j} c^{2} \rho}{2 \lambda_{j}(2-\varepsilon)} \psi_{j}^{2-\varepsilon} .
$$

The bulk chemical potential can be found from $\mu=$ $\partial_{\rho} f(\rho)$, i.e.,

$$
\mu=\theta(\ln \rho+1)+\sum_{j=1}^{n} \frac{G_{j} c^{2}}{2}\left(\frac{\psi_{j}^{2-\varepsilon}}{\lambda_{j}(2-\varepsilon)}+\frac{\psi_{j}^{2}}{\rho}\right) .
$$

Therefore, the free energy is

$$
H=f-\sum_{j=1}^{n} \frac{G_{j} c^{4}}{8 \lambda_{j}(1-\varepsilon)} \frac{\rho\left(\psi_{j}^{\prime}\right)^{2}}{\psi_{j}^{\varepsilon}}\left(\partial_{x} \rho\right)^{2} .
$$

\section{APPENDIX B}

Tables III $-\mathrm{V}$ show the parameters we have used to fit the MPI EOS onto the VW and CS EOS. The fitting has been performed around the two-phase coexistence region. These tables are brought, mainly, to show the capability of the MPI in representing EOSs. Therefore, these values can be used directly to achieve the mentioned systems or utilized for an

TABLE IV. Parameters at which the MPI follows the CS EOS with $a=1$ and $b=4$ for $n=3$.

\begin{tabular}{lcccccc}
\hline \hline$T_{R}$ & 0.78 & 0.82 & 0.86 & 0.90 & 0.94 & 0.98 \\
$\varsigma \times 10^{3}$ & 424.8 & 444.4 & 459.0 & 471.3 & 472.4 & 749.6 \\
$G_{1} \times 10^{3}$ & -126 & -101 & -86.1 & -75.8 & -88.1 & -78.9 \\
$G_{2} \times 10^{3}$ & -845 & -699 & -588 & -497 & -436 & -333 \\
$G_{3}$ & 156.1 & 114.6 & 85.03 & 64.40 & 36.69 & 577.3 \\
$\lambda_{1} \times 10^{3}$ & 61.35 & 56.85 & 55.00 & 54.40 & 64.45 & 33.18 \\
$\lambda_{2} \times 10^{3}$ & 196.8 & 187.7 & 181.1 & 175.6 & 183.4 & 168.1 \\
$\lambda_{3}$ & 1.126 & 1.071 & 1.015 & 0.963 & 0.864 & 1.289 \\
$R^{2}$ & 1.0 & 1.0 & 0.999 & 0.999 & 1.0 & 0.999 \\
\hline \hline
\end{tabular}


TABLE V. Parameters at which the MPI follows the CS EOS with $a=5.5$ and $b=4$ for $n=3$.

\begin{tabular}{lcccccc}
\hline \hline$T_{R}$ & 0.78 & 0.82 & 0.86 & 0.90 & 0.94 & 0.98 \\
$\varsigma$ & 0.991 & 1.015 & 1.076 & 1.104 & 1.102 & 1.132 \\
$G_{1} \times 10^{3}$ & -129.5 & -122.2 & -86.0 & -76.54 & -93.68 & -88.49 \\
$G_{2} \times 10^{3}$ & -850 & -722 & -588 & -498 & -439 & -377 \\
$G_{3}$ & 155.7 & 108.5 & 85.38 & 64.06 & 34.58 & 20.0 \\
$\lambda_{1} \times 10^{3}$ & 62.65 & 65.00 & 55.00 & 54.8 & 66.95 & 68.0 \\
$\lambda_{2} \times 10^{3}$ & 198 & 195.7 & 181.1 & 176.1 & 186.6 & 186.3 \\
$\lambda_{3}$ & 1.124 & 1.051 & 1.016 & 0.962 & 0.854 & 0.777 \\
$R^{2}$ & 1.0 & 1.0 & 0.999 & 0.999 & 1.0 & 1.0 \\
\hline \hline
\end{tabular}

initial guess of curve fittings for other thermodynamic states including other EOSs or experimental data (the interested reader can contact the authors for the details). Here $R^{2}$ is the coefficient of determination of the curve fittings. Four potentials are found suitable for the VW EOS and three for the CS EOS. It should be noted that for the VW EOS $\varsigma$ is fixed at the system temperature, whereas it is a free parameter for the CS EOS.

\section{APPENDIX C}

Following the discussion in Sec. III H, the system of linear equations of the preset MPI is derived by setting the lattice temperature at fluid temperature $\theta=T$, i.e., $\varsigma=\sqrt{3 T}$ and imposing the desired conditions on the MPI EOS (23): The saturation pressure,

$$
p_{\text {sat }}=\rho_{v} T+\sum_{j=1}^{5} \frac{3 T G_{j}}{2} \exp \left(-2 \lambda_{j} / \rho_{v}\right),
$$

the sound speed in the vapor phase,

$$
(\partial p / \partial \rho)_{v}=T+\sum_{j=1}^{5} \frac{3 T \lambda_{j} G_{j}}{\rho_{v}^{2}} \exp \left(-2 \lambda_{j} / \rho_{v}\right),
$$

the sound speed in the liquid phase,

$$
(\partial p / \partial \rho)_{l}=T+\sum_{j=1}^{5} \frac{3 T \lambda_{j} G_{j}}{\rho_{l}^{2}} \exp \left(-2 \lambda_{j} / \rho_{l}\right),
$$

the Maxwell equal-area rule (27),

$$
\begin{aligned}
p_{\text {sat }} & \left(\frac{1}{\rho_{v}}-\frac{1}{\rho_{l}}\right)-T \ln \left(\rho_{l} / \rho_{v}\right) \\
= & \sum_{j=1}^{5} \frac{3 T G_{j}}{4 \lambda_{j}}\left[\exp \left(-2 \lambda_{j} \rho_{l}\right)-\exp \left(-2 \lambda_{j} / \rho_{v}\right)\right],
\end{aligned}
$$

and the boundary condition $p\left(\rho_{l}\right)=p\left(\rho_{v}\right)$,

$$
\begin{aligned}
& \rho_{v} T+\sum_{j=1}^{5} \frac{3 T G_{j}}{2} \exp \left(-2 \lambda_{j} / \rho_{v}\right) \\
& =\rho_{l} T+\sum_{j=1}^{5} \frac{3 T G_{j}}{2} \exp \left(-2 \lambda_{j} / \rho_{l}\right) .
\end{aligned}
$$

If we set $\lambda_{1}=m \rho_{c}$ and $\lambda_{j}=4^{j-1} \lambda_{1},\left\{G_{j}: j=1-5\right\}$ are the unknowns which can be found by solving this system of five linear equations and using the data listed in Table II for the case of this study. Therefore, the MPI forces are determined and can be implemented in the LB system to achieve the desired two-phase system.
[1] A. K. Gunstensen, D. H. Rothman, S. Zaleski, and G. Zanetti, Phys. Rev. A 43, 4320 (1991).

[2] X. Shan and H. Chen, Phys. Rev. E 47, 1815 (1993).

[3] X. Shan and H. Chen, Phys. Rev. E 49, 2941 (1994).

[4] M. R. Swift, W. R. Osborn, and J. M. Yeomans, Phys. Rev. Lett. 75, 830 (1995).

[5] M. R. Swift, E. Orlandini, W. R. Osborn, and J. M. Yeomans, Phys. Rev. E 54, 5041 (1996).

[6] X. He, X. Shan, and G. D. Doolen, Phys. Rev. E 57, R13 (1998).

[7] L.-S. Luo, Phys. Rev. Lett. 81, 1618 (1998).

[8] X. He and G. Doolen, J. Stat. Phys. 107, 309 (2002).

[9] A. L. Kupershtokh, D. A. Medvedev, and D. I. Karpov, Comput. Math. Appl. 58, 965 (2009).

[10] J. Hyväluoma and J. Harting, Phys. Rev. Lett. 100, 246001 (2008).

[11] M. Sbragaglia, R. Benzi, L. Biferale, H. Chen, X. Shan, and S. Succi, J. Fluid Mech. 628, 299 (2009).

[12] X. Shan, Phys. Rev. E 73, 047701 (2006).

[13] J. Bao and L. Schaefer, Appl. Math. Model. 37, 1860 (2013).

[14] Z. Yu and L.-S. Fan, J. Comput. Phys. 228, 6456 (2009).

[15] C. Pan, M. Hilpert, and C. T. Miller, Water Resour. Res. 40 W01501 (2004).
[16] R. Benzi, L. Biferale, M. Sbragaglia, S. Succi, and F. Toschi, Phys. Rev. E 74, 021509 (2006).

[17] S. Succi, The Lattice Boltzmann Equation: For Fluid Dynamics and Beyond (Oxford University Press, Oxford, 2001).

[18] M. Sbragaglia, R. Benzi, L. Biferale, S. Succi, K. Sugiyama, and F. Toschi, Phys. Rev. E 75, 026702 (2007).

[19] X. Shan, Phys. Rev. E 77, 066702 (2008).

[20] L. Chen, Q. Kang, Y. Mu, Y.-L. He, and W.-Q. Tao, Int. J. Heat Mass Transf. 76, 210 (2014).

[21] M. Sbragaglia and X. Shan, Phys. Rev. E 84, 036703 (2011).

[22] M. Sbragaglia and D. Belardinelli, Phys. Rev. E 88, 013306 (2013).

[23] P. Yuan and L. Schaefer, Phys. Fluids 18, 042101 (2006).

[24] J. Zhang and F. Tian, Europhys. Lett. 81, 66005 (2008).

[25] Q. Li, K. H. Luo, and X. J. Li, Phys. Rev. E 86, 016709 (2012).

[26] G. Falcucci, G. Bella, G. Shiatti, S. Chibbaro, M. Sbragaglia, and S. Succi, Commun. Comput. Phys. 2, 1071 (2007).

[27] G. Falcucci, S. Chibbaro, S. Succi, X. Shan, and H. Chen, Europhys. Lett. 82, 24005 (2008).

[28] G. Falcucci, S. Ubertini, and S. Succi, Soft Matter 6, 4357 (2010).

[29] S. Chibbaro, G. Falcucci, G. Chiatti, H. Chen, X. Shan, and S. Succi, Phys. Rev. E 77, 036705 (2008). 
[30] R. Benzi, M. Bernaschi, M. Sbragaglia, and S. Succi, Europhys. Lett. 91, 14003 (2010).

[31] H. Huang, M. Krafczyk, and X. Lu, Phys. Rev. E 84, 046710 (2011).

[32] C. E. Colosqui, G. Falcucci, S. Ubertini, and S. Succi, Soft Matter 8, 3798 (2012).
[33] Q. Li, K. H. Luo, and X. J. Li, Phys. Rev. E 87, 053301 (2013).

[34] D. Wolf-Gladrow, Lattice Gas Cellular Automata and Lattice Boltzmann Models (Springer, Berlin/Heidelberg, 2000), pp. 159.

[35] X. Shan, X.-F. Yuan, and H. Chen, J. Fluid Mech. 550, 413 (2006).

[36] Z. Guo, C. Zheng, and B. Shi, Phys. Rev. E 65, 046308 (2002). 